\title{
Seminare 2011
}

\section{Praxiseröffnung/-übernahme}

\section{Themen}

Juristische Aspekte (Praxisbewilligung, Zulassung zur Sozialversicherung, Vertragswesen), Gesellschaftsformen / Ehe- und Erbrecht (Trennung Privat- vom Geschäftsvermögen, Ehegüterstand, Erbschaftsplanung), Praxiseinrichtung (Inneneinrichtung, Kostenberechnung), Praxisadministration (Leistungserfassungs- und Abrechnungssysteme), Unternehmensbewertung einer Arztpraxis (Berechnung und Beurteilung des Unternehmenswertes), Finanzierung der Arztpraxis (Businessplan, Kredite, Absicherungsmöglichkeiten), Versicherungen/Vorsorge/Vermögen (Personen- und Sachversicherungen, Vorsorgeplanung)

\section{Sponsoren}

Die Kosten werden durch diverse Sponsoren (siehe www.fmhservices.ch) gedeckt.

\section{Daten}

\begin{tabular}{|c|c|}
\hline K04 & Donnerstag, 1. September 2011 FMT \\
\hline & 9.00-16.30 Uhr Zürich \\
\hline K05 & $\begin{array}{l}\text { Donnerstag, 3. November } 2011 \text { Hotel Victoria } \\
\begin{array}{ll}9.00-16.30 ~ U h r & \text { Basel }\end{array}\end{array}$ \\
\hline
\end{tabular}

\section{Praxisübergabe}

\section{Themen}

Juristische Aspekte (Praxisübergabevertrag, allg. Vertragswesen, Übergabe der Krankengeschichten), Unternehmensbewertung einer Arztpraxis (Berechnung Inventarwert und Goodwill als Verhandlungsbasis), Versicherungen/Vorsorge/Vermögen (Übergabe/Auflösung von Versicherungsverträgen, Pensions- und Finanzplanung), Steuern (Steueraspekte bei der Praxisübergabe, Optimierung der steuerlichen Auswirkungen, Liquidations- und Grundstückgewinnsteuer, Bestimmung des optimalen Übergabezeitpunktes).

\section{Sponsoren}

Die Kosten werden durch diverse Sponsoren (siehe www.fmhservices.ch) gedeckt.

\section{Daten}

K09 Donnerstag, 8. September 2011 FMT 13.30-18.00 Uhr

Zürich

K10 Donnerstag, 10. November 2011 Hotel Victoria 13.30-18.00 Uhr Basel

\section{Finanz- und Steuerplanung}

\section{Themen}

Finanzplanung (Businessplan, Buchhalterische Massnahmen vor Praxiseröffnung/-übernahme, Standardkontenplan für Ärzte, System der doppelten Buchhaltung, EDV-unterstützte Buchführungslösung), Steuern (Steueraspekte bei Eintritt in die Selbständigkeit, Steuerfallen und Steuerrisiken, optimierte Steuerplanung).

\section{Sponsoren}

Die Kosten werden durch diverse Sponsoren (siehe www.fmhservices.ch) gedeckt.

\section{Datum}

K12 Donnerstag, 15. September 2011 Schmiedstube 13.30-18.00 Uhr Bern

\section{Tarifwerk TARMED - Einführungskurs}

Das Seminar richtet sich an Ärztinnen und Ärzte mit bestehender Praxis und an solche, die kurz vor einer Praxiseröffnung oder Praxisübernahme stehen. Der Einführungskurs vermittelt den Kursteilnehmern die Grundlagen des Tarifwerkes TARMED. Die Seminarteilnehmerzahl ist begrenzt.

\section{Themen}

Fakten (Gesetzliche und vertragliche Grundlagen), Struktur (Tarifbrowser, Grundstruktur des Tarifwerkes, Regelhierarchie, Leistungsblöcke, Leistungsgruppen), Generelle Interpretationen (Wichtigste generellen Interpretationen), Parameter einer Tarifposition (Alle Parameter einer Tarifposition), Tarifpositionen aus dem Kapitel 00 Grundleistungen (Diverse Tarifpositionen aus dem Kapitel 00 Grundleistungen), Praxislabor und Präsenzdiagnostik (Neue Analyseliste), Organisationen und Informationsquellen (www.tarmedsuisse.ch usw.).

\section{Kosten}

200 CHF (inkl. Kursunterlagen).

\section{Datum}

K61

$\begin{array}{ll}\text { Dienstag, 20. September } 2011 & \begin{array}{l}\text { Hotel Arte } \\ \text { Olten }\end{array}\end{array}$

\section{Praxismarketing für Ärzte}

Das Seminar richtet sich an Ärztinnen und Ärzte, welche vor einer Praxiseröffnung stehen oder bereits eine Praxis führen. 


\section{Themen}

Der Erfolg einer Arztpraxis ist unlösbar mit gutem Service und Kundenorientierung verbunden. Damit sind mehr als gute Umgangsformen gemeint. Echter Service geht tiefer und bietet den Patientinnen und Patienten - oder Kunden - einen direkten Nutzen. Aus einer Arztpraxis wird DIE Praxis.

Die Herausforderung ist nicht, Service für Patienten zu leisten, sondern Service für Patienten aus der Sicht des Patienten erlebbar zu machen. Dazu gehört, dass man seine Kunden versteht, auf ihre Bedürfnisse vorbereitet ist, sie um Informationen bittet, ihnen genau zuhört, Verantwortung für das eigene Tun übernimmt, über das normale Mass hinaus engagiert zu sein, Menschen zu überraschen, und das immer regelmässig und auf gleichbleibend hohem Niveau!

Das Wichtigste zu den Themen Telefon, Empfang, Teamentwicklung wird diskutiert. Die wichtigsten Werbemöglichkeiten besprochen. Nicht das Aussergewöhnliche wollen wir tun, sondern das Gewöhnliche aussergewöhnlich gut!

Kosten

300 CHF (inkl. sämtlicher Kursunterlagen und Verpflegungen).

\section{Seminarsponsoren 2011}

Die Unterstützung durch verschiedene Sponsoren ermöglicht es der FMH Consulting Services AG, ihre Seminarreihen für FMH-Mitglieder teils kostenlos, teils kostengünstig anzubieten. Gerne stellen wir Ihnen diese Firmen in einem Kurzporträt vor.

\section{medics labor \\ professionell und persönlich}

Medics Labor AG

Chutzenstrasse 24, 3001 Bern

Tel. 03137220 02, Fax 0313714044

info@medics-labor.ch

www.medics-labor.ch

\section{Medizinisches Labor und mehr}

Medics Labor ist ein Schweizer Unternehmen, zu Hause in Bern, hier verwurzelt und seit vielen Jahren erfolgreich tätig im Kanton sowie weiteren Regionen. Geschätzt als persönliches, unkompliziertes Gegenüber, überzeugt Medics Labor durch fachliches und menschliches Gespür mit zahlreichen Hilfestellungen und Dienstleistungen. Wir verstehen uns als sozialer Arbeitgeber und beschäftigen auch behinderte Personen.

Medics Labor ist ein Labor von Ärzten für Ärzte. Es gehört den Laborspezialisten und den Ärzten, die das Unternehmen gemeinsam führen.

\section{Datum}

K64

Mittwoch, 7. September 2011 09.00-16.30 Uhr

Schmiedstube Bern

\section{Anmeldung und Auskunft}

www.fmhservices.ch oder FMH Consulting Services, Cornelia Steinmann, Burghöhe 1, 6208 Oberkirch, Tel. 04192500 77, Fax 0419210586.

\section{Hinweis}

Bei sämtlichen Seminaren, bei denen die Kosten teilweise oder gänzlich von Seminarsponsoren gedeckt werden, werden die Teilnehmeradressen den jeweiligen Sponsoren zur Verfügung gestellt.

\section{Annullierungsbedingungen}

Bei Abmeldungen oder Fernbleiben werden folgende Unkostenbeiträge erhoben:

- 50 CHF pro Person ab 14 Tage vor Seminarbeginn; - 100 CHF pro Person ab 7 Tage vor Seminarbeginn oder Fernbleiben.

\section{s్gొ}

Bioanalytica AG

Maihofstrasse 95a, 6006 Luzern

Tel. 04142931 31, Fax 0414293130

service@bioanalytica.ch

www.bioanalytica.ch

\section{Engagierte Kompetenz}

Bioanalytica, 1957 in Luzern gegründet, basiert auf einer langjährigen Tradition. Stetige Innovation und ein Team qualifizierter Fachspezialisten und Labormediziner bilden das Fundament unserer Kompetenz. Qualität und Seriosität - das sind die Werte, denen wir uns verschrieben haben. Aus der Überzeugung, dass dies auch unseren Kunden wesentliche Vorteile bietet, haben wir unser Labor im Jahre 2000 akkreditieren lassen.

Schnell, wenn notwendig auch rund um die Uhr, sind wir für Sie da. Mit dem Know-how von rund 85 Mitarbeitenden, modernster Laborautomation und Informationstechnologie sind unsere Laborresultate in kürzester Zeit verfügbar.

Bei Bioanalytica stehen Sie als Kunde im Mittelpunkt. Wir unterstützen Sie und Ihr Praxisteam jederzeit gerne optimal im persönlichen Kontakt und mit zahlreichen wertvollen zusätzlichen Dienstleistungen. 


\section{$\{$ ANALYTICA \\ MEDIZINISCHE LABORATORIEN AG}

Analytica Medizinische Laboratorien AG

Falkenstrasse 14

8024 Zürich

Tel. 0442505050

kundendienst@analytica.ch

\section{Werte. Verbinden.}

Sehr geehrte Frau Kollega, sehr geehrter Herr Kollege Die Analytica Medizinische Laboratorien AG wurde 1957 von meinem Vater gegründet, und ich durfte das Labor 1985 in zweiter Generation übernehmen. Eigentlich mag ich es gar nicht, mich unpersönlich und mit schönen Worten vorzustellen. Ich bin durch und durch Praktiker, gibt es ein Problem, dann löse ich es, und kennt mich ein Kunde noch nicht persönlich, dann komme ich gerne vorbei und stelle mich vor.

Wir haben uns nach langen Diskussionen für den Slogan «Werte. Verbinden.» entschieden. Der Punkt zwischen den Worten ist kein Schreibfehler. Wir haben Werte. Werte, die uns einen persönlichen Umgang miteinander ermöglichen, Werte, die die Qualität unserer Arbeit beschreiben und Werte, die wir mit Ihnen - unseren Kunden- teilen. Diese Werte verbinden uns miteinander und stellen das tragfähige Netz dar, das uns alle seit so vielen Jahren trägt. Für das bin ich dankbar.

\section{$\mathrm{S}_{3}$ polyanalytic}

Polyanalytic SA

Avenue de Sévelin 18, 1004 Lausanne

Tel. 02180492 50, Fax 0218049250

info@polyanalytic.ch

www.polyanalytic.ch

Polyanalytic ist ein Labor für medizinische Analysen, das auf dem Gebiet der Kantone Waadt und Neuenburg tätig ist.

Gestützt auf seine Kompetenzen, die es in den Dienst der Patientinnen und Patienten und der Ärzteschaft stellt, bietet Polyanalytic eine umfassende Palette von medizinischen Analysen.

Seit Polyanalytic 1983 gegründet wurde, ist das Unternehmen für herausragende Qualität und kundennahe Dienstleistungen bekannt. Den frei praktizierenden Ärztinnen und Ärzten werden mit unvergleichlicher Konstanz verlässliche, rasche und kompetente Leistungen geboten, damit sie ihre Kunst ausüben können.

Mit Polyanalytic verfügen die Ärztinnen und Ärzte nicht nur über einen Partner, der auf ihre Bedürf- nisse eingeht, sondern auch tagtäglich über echte Unterstützung bei ihrer Tätigkeit.

Polyanalytic ist mehr als ein Unternehmen: Dank der Kompetenz der Menschen, die dort arbeiten, kann die Ärzteschaft darauf vertrauen, dass bei den Patientinnen und Patienten, für die es verantwortlich ist, optimale Laborkontrollen gewährleistet sind.

\section{s్jొ dianalabs}

Dianalabs SA

Rue de la Colline 6, 1205 Genève

Tel. 02280712 40, Fax 0228071244

info@dianalabs.ch

www.dianalabs.ch

Dianalabs ist ein Labor für medizinische Analysen, das 1988 mit dem Ziel gegründet wurde, der Ärzteschaft und den Patientinnen und Patienten optimale Laborkontrollen zu bieten.

Aufgrund seiner Publikationen und Präsentationen ist das Genfer Labor für die Qualität seiner Serologie international anerkannt.

Wir bieten eine umfassende Palette von medizinischen Analysen, um alle Bedürfnisse der Medizin abzudecken. Doch Dianalabs ist mehr als ein polyvalentes Allround-Labor: Dank seinem Spezialistenteam deckt es eine Vielzahl von Fachgebieten ab und bietet eine Schnittstelle $\mathrm{zu}$ jedem medizinischen Fachgebiet mit seinen besonderen Bedürfnissen.

Durch die wissenschaftliche Zusammenarbeit mit den Ärztinnen und Ärzten und den universitären Zentren wurde uns klar, dass nur ein regionales Unternehmen, das grundlegende menschliche Werte wie Qualität, Austausch und Dienstleistungsbereitschaft in den Vordergrund stellt und mit den lokalen Verhältnissen vertraut ist, die Bedürfnisse der Bevölkerung und der Ärzteschaft kompetent erfüllen kann.

\section{Schmid MOGELSBERG}

Schmid Mogelsberg AG, Ärztedrucksachen Sonnmattstrasse 1, 9122 Mogelsberg Tel. 07137560 80, Fax 0713756081 info@schmid-mogelsberg.ch www.schmid-mogelsberg.ch

\section{Seit über 70 Jahren auf Ärztedrucksachen spezialisiert!}

Bei der Gestaltung von zweckmässigen Arztformularen (Patientenkarten, KG-Einlagenblätter usw.) profitieren Sie von der langjährigen Erfahrung. Die Vergangenheit hat gezeigt, dass sich kaum zwei Ärzte 
für den gleichen Druck entscheiden. Zweckmässige Materialauswahl, einwandfreie Verarbeitung, freundliche und kompetente Beratung, schnelle Lieferung und die Ausführung von Spezialwünschen - diese Dienstleistungen schaffen die Grundlage für ein langjähriges Vertrauensverhältnis. Auf Wunsch versenden wir eine individuell auf Ihre Fachrichtung zusammengestellte Druckmusterkollektion.

An über 9000 Ärzte liefern wir Drucksachen, Papiere und Büroartikel für den Praxisalltag.

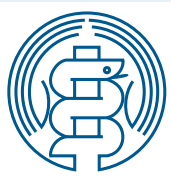

Schweizerische Ärzte-Krankenkasse

Oberer Graben 37, Postfach 2046, 9001 St. Gallen

Tel. 07122718 18, Fax 0712271828

info@saekk.ch

www.saekk.ch

Die richtige Adresse für Erwerbsausfalldeckungen, Kollektivkrankenkasse und Versicherungsplanung

Mit mehr als 100 Jahren Erfahrung kennt unsere Organisation auch heute die Bedürfnisse der Ärztinnen und Ärzte. Sie bietet entsprechend durchdachte und kostengünstige Lösungen an, sowohl für Praxiseröffner/innen wie auch für selbständige und angestellte Ärztinnen und Ärzte.

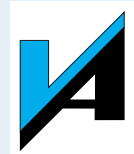

Versicherung der Schweizer Ärzte Genossenschaft Länggassstrasse 8, 3000 Bern 9

Tel. 03130125 55, Fax 0313025156

versa@versa.ch

www.versa.ch

Spezialisiert auf die Bedürfnisse von Ärztinnen und Ärzten und deren Ehegatten, bietet die Versicherung der Schweizer Ärzte Genossenschaft individuelle, den jeweiligen Bedürfnissen angepasste Versicherungslösungen im Bereich der privaten Vorsorge an.

\section{Unilabs}

www.unilabs.ch

UNILABS - Ihr Qualitätslabor

schnell, zuverlässig und nah

Unilabs ist im Bereich der medizinischen Analysen ein kompetenter, transparenter und zuverlässiger
Partner. Wir bieten Ihnen überall in der Schweiz ein komplettes Analysenspektrum, umfassende Dienstleistungen und kompetente Fachberatung an. In der Deutschschweiz sind dies Unilabs Mittelland mit den Standorten Basel, Bern, Langnau, Solothurn, Thun; Unilabs Zürich und Unilabs Dr. Weber.

Unilabs bietet Weiterbildungen für das gesamte Praxis-Team sowie Beratung bei Praxis- und Laborbedarf.

Unsere vielseitigen und regionalen Dienstleistungen basieren auf einer fundierten wissenschaftlichen Struktur und hochstehenden Qualität.

Unilabs Schweiz - aktuell 900 Mitarbeiterinnen und Mitarbeiter, 72 Wissenschaftler und 22 Labors in Ihrer Nähe.

\section{EMH $\underset{\text { SChWABE }}{\text { FMH }}$ \\ Editores Medicorum Helveticorum}

EMH Schweizerischer Ärzteverlag AG Farnsburgerstrasse 8, 4132 Muttenz Tel. 06146785 55, Fax 0614678556 verlag@emh.ch www.emh.ch

EMH, der Verlag der Ärztinnen und Ärzte Der Verlag EMH Schweizerischer Ärzteverlag AG wurde 1997 gegründet. EMH ist ein Gemeinschaftsunternehmen der Verbindung der Schweizer Ärztinnen und Ärzte FMH und der Schwabe AG, Basel, dem mit Gründung 1488 ältesten Druck- und Verlagshaus der Welt.

Hauptpublikationen von EMH sind die Zeitschriften «Schweizerische Ärztezeitung», das offizielle Publikationsorgan der FMH, «Swiss Medical Forum» mit praxisorientierten Fortbildungsbeiträgen, sowie «Swiss Medical Weekly», die Plattform für klinisch orientierte Wissenschaftler. Ebenfalls zu den Hauptpublikationen zählt «PrimaryCare», die offizielle «Schweizerische Zeitschrift für Hausarztmedizin».

Als erfolgreiches Online-Angebot ist unter anderem die Fortbildung des «Swiss Medical Forum» unter www.smf-cme.ch zu nennen. Steigende Zugriffszahlen und die Akkreditierung durch die Fachgesellschaften SGAM und SGIM als strukturierte und nachweisbare Fortbildung belegen diesen Erfolg. Weitere medizinische Fachzeitschriften, ein ständig wachsendes Buchprogramm sowie viele Kooperationen und Dienstleistungen runden das umfangreiche Verlagsangebot ab. 


\section{medica}

\section{MEDIZINISCHE LABORATORIEN Dr. F. KAEPPEUIAG}

MEDIZINISCHE LABORATORIEN DR. F. KAEPPELI AG

Eidgenössisch anerkannte Laboratorien

Wolfbachstrasse 17, 8024 Zürich

Tel. 04426999 99, Fax 0442699909

info@medica-labor.ch

www.medica-labor.ch

Der promovierte Mikrobiologe und Biochemiker Dr. F. Käppeli, Laborspezialist FAMH, übernahm 1976 das heute über 50-jährige Unternehmen und gründete als dessen Leiter und Inhaber die Einzelfirma medica. Der wichtigste unternehmerische Leitgedanke von Dr. F. Käppeli heisst kontinuierliche Innovation und Schaffung wegweisender Standards auf allen Gebieten der Labormedizin: Mikrobiologie inklusive Parasitologie, Serologie, Immunologie, klinische Chemie, Hämatologie, molekulare Diagnostik und Pathologie in Human- und Veterinärmedizin. So entstand ein Kompetenz-Zentrum für Labordiagnostik von gesamtschweizerisch grosser Bedeutung. Die modernst ausgebauten Laboratorien werden laufend erweitert und befinden sich im Herzen von Zürich. Über 200 Angestellte der Partnerlabors, begleitet von Spezialisten aus Medizin, Pharmakologie, Naturwissenschaften und Technik, garantieren für höchste Professionalität.

\section{IVF HARTMANN AG}

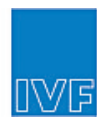

\section{IVF HARTMANN AG}

Victor-von-Bruns-Strasse 28, 8212 Neuhausen

Tel. 05267431 11, Fax 0526727441

info@ivf.hartmann.info

www.ivf.hartmann.info

Die IVF HARTMANN AG ist einer der führenden Anbieter für medizinische Verbrauchsgüter im Bereich Heilung, Pflege und Hygiene in der Schweiz. Ihre Lösungen helfen überall dort, wo Menschen geholfen wird. Zu ihren Kunden zählen somit Spitäler, Alters- und Pflegeheime, Spitex-Organisationen, niedergelassene Ärzte, Apotheken, Drogerien und der Lebensmitteleinzelhandel. Das breite Angebot der IVF HARTMANN AG umfasst über 2000 Produkte vom therapeutisch wirksamen Pflaster (z.B. Isola ${ }^{\circledR}$ Capsicum N) über funktionelle Verbände bis hin zu Produkten für die moderne Wundbehandlung (z.B. TenderWet $^{\circledR}$ oder CompriGel $^{\circledR}$ ) und Erste Hilfe (z.B. DermaPlast $^{\circledR}$ ). Die IVF HARTMANN GRUPPE ist eine 60-prozentige Tochtergesellschaft der PAUL HARTMANN AG mit Sitz in Heidenheim an der Brenz (D) und beschäftigt rund 350 Mitarbeiter. Neben ihrem Hauptsitz in Neuhausen am Rheinfall (SH) verfügt die IVF HARTMANN AG über weitere Produktionsstätten in Gommiswald (SG) und Netstal (GL).

\section{mepha}

Mepha Pharma AG

Dornacherstrasse 114, 4147 Aesch

Tel. 0617054343

www.mepha.ch

\section{Mepha - wir setzen Massstäbe}

Mepha, die führende Generika-Herstellerin der Schweiz, steht im 7. Jahrzehnt ihrer denkwürdigen Erfolgsgeschichte. Unseren Beitrag zu wirksamer Prophylaxe und Therapie sehen wir in der Entwicklung, Produktion und Vermarktung von günstigen, gut verträglichen und hochwertigen Generika. Wir entwickeln und produzieren in der Schweiz mit modernsten Hightechverfahren und nach höchstem Schweizer Qualitätsstandard. Unsere innovativen, kreativen Lösungen begeistern unsere Kunden immer wieder aufs Neue: Zum Beispiel neuartige und verbesserte Anwendungsformen unserer Medikamente, die den Behandlungserfolg und das Wohlbefinden von Patientinnen und Patienten steigern. Alle unsere Leistungen gründen auf einer ganzheitlichen Sicht, welche die Interessen unserer Kunden, Mitarbeiter und Aktionäre, aber auch jene der übrigen Anspruchsgruppen in den Mittelpunkt stellt. Erstklassige Produkte, ein komplettes Package gefragter Dienstleistungen und offene Kommunikation sind weltweit Basis der Zufriedenheit unserer Kunden.

\section{$\int \begin{aligned} & \text { alzmann } \\ & M \text { E D I C O }\end{aligned}$}

Salzmann AG

Salzmann MEDICO

Rorschacher Strasse 304, 9016 St. Gallen

Tel. 07128212 12, Fax 0712821210

medico.sg@salzmann-group.ch

www.salzmann-group.ch

Salzmann MEDICO wurde 1980 durch Herrn Daniel Künzli, Präsident der Salzmann Group, gegründet. Die sehr kundenorientierte Handelsfirma vertreibt medizinische Verbrauchsgüter und Einwegprodukte. Die innovativen medizinischen Kompressionsstrümpfe der Marken VENOSAN ${ }^{\circledR}$ und VENOFIT ${ }^{\circledast}$ aus der Produktion von Salzmann MESH werden weltweit exportiert.

Produktesortiment: Produkte aus Produktion der Salzmann Abteilung MESH Marke VENOSAN ${ }^{\circledR}$; Exklusiv-Vertretungen unter Original-Markennamen; Private Label Produkte (SAMA ${ }^{\circledR}$, SAMA Orthopaedics ${ }^{\circledR}$, Tale $^{\circledR}$, Thermoban $\left.{ }^{\circledR}\right)$; Wundkompressen; Wundtupfer; diverse Verbandsmaterialien; Heftpflaster / Wundschnellverbände; elastische Binden; medizinische Kompressionsstrümpfe / Stützstrümpfe; Körperban- 
dagen, Orthesen, Schienen; Chirurgisches Nahtmaterial; Fixationsprodukte (Gips / synthetische Steifverbände); OP-Handschuhe; OP-Abdeckungen / OP-Bekleidung; OP-Sets steril; Produkte für die Sterilisation und Sterilisations-Kontrolle; Inkontinenzprodukte.

\section{EGalexis}

Galexis AG

Industriestrasse 2, Postfach, 4704 Niederbipp

Tel. 05885171 11, Fax 0588517114

info@galexis.com

www.galexis.com

Als Vollgrossist setzt Galexis AG Standards im Schweizer Gesundheitsmarkt. Wir beliefern unsere Kunden ganz nach dem Motto «Alles aus einer Hand» mit Pharma, Praxis- und Laborbedarf sowie Medizintechnik und erbringen darüber hinaus integrierte Dienstleistungen in der Gesundheitslogistik - schweizweit. Mit erprobten Lösungen fördert Galexis den Erfolg ihrer Kunden.

Möchten Sie ausserdem Ihre eigene Praxis praktisch, funktionell und ästhetisch einrichten? Genau hier kann Sie Galexis mit ihren Fachpartnern und einer langjährigen Erfahrung professionell beraten und unterstützen!

Überzeugen Sie sich - mit Galexis können Sie rechnen!

\section{MSD}

MSD Merck Sharp \& Dohme-Chibret AG

Schaffhauserstrasse 136, 8152 Opfikon-Glattbrugg

Tel. 04482871 11, Fax 0448287210

www.msd.ch

www.univadis.ch

MSD ist die Schweizer Niederlassung von Merck \& Co., Inc. Whitehouse Station mit Hauptsitz in New Jersey, USA.

\section{DEM PATIENTEN VERPFLICHTET.}

Das Wohl des Patienten steht in unserer täglichen Arbeit an erster Stelle.

Als weltweit tätiger, forschender Arzneimittelhersteller entwickeln, produzieren und vertreiben wir innovative Medikamente und Impfstoffe. Wir tun dies seit mehr als 100 Jahren und heute in über 20 Therapiegebieten.

In unserer Verpflichtung dem Patienten gegenüber ermöglichen wir weltweit die Versorgung mit dringend benötigten Medikamenten und unterstützen nachhaltige Gesundheitsprogramme vor Ort.



Helvepharm AG

Walzmühlestrasse 48, 8500 Frauenfeld

Tel. 05272328 50, Fax 0527232858

info@helvepharm.ch

www.helvepharm.ch

Als Vermarkter und Vertreiber hochwertiger Generika tun wir alles dafür, um mit Innovationsgeist und Qualität eine führende Rolle im Schweizer Markt zu erreichen.

Helvepharm setzt auf preiswerte Generika. Auf Medikamente, die sich in Wirkstoff, Darreichungsform und Dosierung an die Originalpräparate anlehnen und mit diesen austauschbar sind. Auch unsere Arzneimittel werden durch Swissmedic auf Herz und Nieren geprüft. Helvepharm Generika sind gleich wirksam wie das Original, jedoch ungleich günstiger. Mit über 60 Wirkstoffen in über 330 Darreichungsformen bieten wir kluge Alternativen zu Antihypertonika, Antidepressiva, Lipidsenkern, Gastrotherapeutika und für weitere relevante Gebiete. Helvepharm ist die günstige Basis im Gesundheitswesen. Helvepharm in Frauenfeld gehört zu Zentiva und damit zum drittgrössten Generikaanbieter in Europa. Zentiva ist Teil der sanofi-aventis-Gruppe.

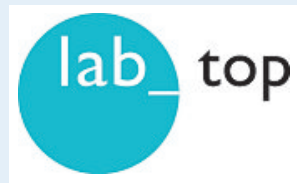

LabTop Medizinische Laboratorien AG Chriesbaumstrasse 6, 8604 Volketswil Tel. 04339930 30, Fax 0433993031 info@labtop.ch www.labtop.ch

\section{Das externe Labor in Ärztehand}

Ärzte halten zusammen: LabTop ist ein von Ärzten aufgebautes und von Labor-Profis geführtes Privatlabor. Seit 10 Jahren unterstützt es den Arzt in seinem Sinne. LabTop ist überwiegend im Besitz von Ärzten und steht beteiligungswilligen Ärzten weiterhin offen.

Zur Selbsthilfe von praktizierenden Ärzten gestartet, klein, modern, unabhängig, exakt - typisch schweizerisch eben - bietet LabTop bestechend einfache und modernste Lösungen für die Arztpraxis. Bei LabTop bleibt die externe Analytik in Ärztehand.

Vorteile für Sie: Als Novum bietet LabTop ein webbasiertes Geschäftsmodell, von dem Sie in verschiedener Hinsicht profitieren: Ressourcen-Einsparungen in Ihrer Praxis dank optimierter Prozesse, top Service, Messqualität, die höchsten Ansprüchen ge- 
nügt, und nicht zuletzt, Ihr Beitrag bei der Analyseerfassung ist LabTop etwas wert.

LabTop - von Ärzten aufgebaut, für Ärzte geführt.

\section{एँम}

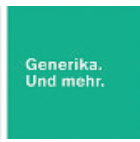

Teva Pharma AG

Industriestrasse 111, 4147 Aesch

Tel. 06175697 50, Fax 0617569755

office@tevapharma.ch

www.tevapharma.ch

Auf den ersten Blick Generika.

Auf den zweiten noch viel mehr.

Teva steht für Generika. Und für noch viel mehr. Denn mit unserer Philosophie, stets mehr zu leisten, sind wir zu einem international aktiven Pharmaunternehmen mit mehreren Standbeinen herangewachsen.

Weil wir Gesundheit nicht nur erhalten, sondern auch erschwinglich machen wollen, stehen wir mittlerweile an der Spitze der weltweit führenden Generika-Hersteller.

Mit diesen Kompetenzen möchten wir Ihnen als verlässlicher Partner auf dem Schweizer Pharmamarkt zur Seite stehen. Dabei bieten wir Ihnen stets mehr: mehr Service, mehr Engagement und mehr Sicherheit und Qualität, welche wir in unseren eigenen Produktionsstätten optimal kontrollieren können. Mehr über uns erfahren Sie unter www.tevapharma.ch

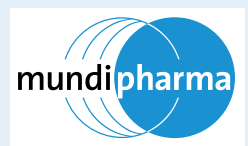

Mundipharma Medical Company

Zweigniederlassung Basel

St. Alban-Rheinweg 74, Postfach, 4020 Basel

Tel. 06120511 11, Fax 0612051187

info@mundipharma.ch

www.mundipharma.ch

Mundipharma Medical Company ist die Schweizer Firma einer mittelgrossen, international erfolgrei- chen Pharmagruppe. Schmerztherapie, Onkologie und Atemwegserkrankungen sind die Kompetenzschwerpunkte unserer Forschung. Wir verstehen uns heute als modernes Dienstleistungsunternehmen, das hochwirksame Arzneimittel mit grösstmöglicher Verträglichkeit entwickelt und somit die Therapie für Arzt und Patient wesentlich erleichtert.

Dieser Anspruch, unsere Arbeit eng an den Bedürfnissen der Menschen auszurichten, fordert uns täglich bei der Suche nach noch besseren Wirkmechanismen neu heraus. Die Motivation, durch hochwirksame Medikamente helfen zu können, ist dabei Ansporn und Herausforderung zugleich.

\section{zur Rose}

Zur Rose

Walzmühlestrasse 60, 8500 Frauenfeld

Tel. Ärztegrossist 0527240020

Tel. Versandapotheke 0848842842

info@zur-rose.ch

www.zur-rose.ch

Zur Rose - zuverlässiger und vertrauensvoller Partner von mehr als 3000 Ärztinnen und Ärzten Zur Rose ist Marktführer in der Schweizer Medikamentendistribution in den Bereichen Medikamentenversand und Arztpraxisbelieferung. Als standeseigenes Unternehmen vertritt Zur Rose die Interessen der Ärzteschaft.

\section{Zur Rose für Ärzte}

Mehr als 3000 Arztpraxen in der ganzen Schweiz erhalten von Zur Rose alles geliefert, was sie täglich benötigen: Medikamente, Impfstoffe, Insuline, Seren, Magistralitäten, Verbrauchsmaterialien, Labor- und Röntgenbedarf sowie Praxisgeräte und Instrumente aller Art.

\section{Zur Rose für Patienten}

Im Auftrag der Ärztinnen und Ärzte verschickt die Versandapotheke Zur Rose portofrei Medikamente an über 200000 Kundinnen und Kunden. Zur Rose führt die Medikamenten- oder Bezugschecks kostenfrei aus und gewährt zusätzlich bis zu 12\% Rabatt. 